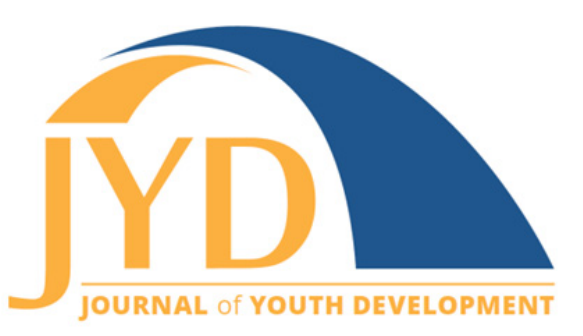

http://jyd.pitt.edu/ | Vol. 12 Issue 4 DOI 10.5195/jyd.2017.521 | ISSN 2325-4017 (online)

\title{
Towards the Promotion of Positive Development among Boys in Challenging Contexts: A Mixed-Methods Study of Engagement in the Scoutreach Initiative
}

\author{
Robey B. Champine \\ Yale University \\ robey.champine@yale.edu
}

Sara K. Johnson

Tufts University

s.johnson@tufts.edu

\begin{abstract}
Engagement in youth development programs reflects the quality of young people's program-related experiences. However, more research is needed that explores cognitive, emotional, and behavioral dimensions of engagement in programs that serve underrepresented youth of color. The present crosssectional and mixed-methods study assessed potential relations among dimensions of engagement in the Boston-area Scoutreach initiative, character attributes, self-perceived school competence, and intentional self-regulation. We analyzed data from 32 Scouts (Mage $=9.97$ years, $S D=2.46$, Range $=6$ to 14 ), 32 parents/guardians, and five Scoutreach leaders. Scouts demonstrated that they were cognitively, emotionally, and behaviorally engaged in Scoutreach, and these dimensions were related differentially to indicators of healthy development. Qualitative data elucidated key aspects of Scoutreach (e.g., camping, peer relationships) that were linked to youth engagement. We discuss limitations of the present study and implications for future research and practice.
\end{abstract}

Key words: youth development programs, youth engagement, character attributes, positive youth development

\section{Introduction}

High-quality youth development (YD) programs (e.g., 4-H, Boy Scouts of America [BSA], Boys \& Girls Clubs) may represent key ecological developmental assets in the lives of diverse youth

\footnotetext{
(c)) EY New articles in this journal are licensed under a Creative Commons Attribution 4.0 License. This journal is published by the University Library System, University of Pittsburgh and is cosponsored by the University of Pittsburgh Press. The Journal of Youth Development is the official peer-reviewed publication of the National Association of Extension 4-H Agents and the National AfterSchool Association.
} 


\section{Engagement in Scoutreach}

(Vandell, Larson, Mahoney, \& Watts, 2015). These programs immerse youth in structured activities in safe and supervised settings with opportunities to enhance their socialization and life skills (Roth \& Brooks-Gunn, 2016). High-quality YD programs emphasize a positive youth development (PYD) perspective, through viewing youth as resources to be developed (Lerner et al., 2005).

Relational developmental systems (RDS) metatheory (Overton, 2015) represents an ideal frame through which to examine how ecological developmental assets, such as YD programs, may optimize the development of diverse youth. This holistic and integrated view of human development emphasizes that all youth have strengths and the capacity for healthy growth that results from mutually-influential, individual-context relations (Overton \& Lerner, 2014). Youth thrive when their individual developmental assets or strengths are connected with ecological developmental assets (Lerner, Agans, DeSouza, \& Gasca, 2013).

Urban youth of color in economically disadvantaged communities are underrepresented in YD programs (e.g., Lee, Borden, Serido, \& Perkins, 2009). However, when they do participate, these youth are likely to benefit, such that YD programs may buffer youth against potential contextual risk factors (Urban, Lewin-Bizan, \& Lerner, 2009). For instance, in a large sample of diverse young people, youth who showed the highest scores on self-regulation were girls from lower-resource environments who participated in YD programs (e.g., Big Brothers/Big Sisters) and other out-of-school-time (OST) activities (Urban et al., 2009). In short, structured activities may serve a protective function in urban, lower-resource neighborhoods (e.g., Francois, Overstreet, \& Cunningham, 2011) by providing a safe space for youth of color who are exposed to violence and other potentially challenging experiences, and may connect them with supportive mentors (Francois et al., 2011).

\section{Key Dimensions of Youth Program Participation}

Research on YD programs has primarily examined young people's quantity of participation, commonly indexed by breadth, duration, and intensity (Bohnert, Fredricks, \& Randall, 2010). In comparison, engagement is a multidimensional construct that reflects the quality of young people's program-related experiences and how youth attribute meaning to their experiences (Tiffany, Exner-Cortens, \& Eckenrode, 2012). More research is needed that examines multiple dimensions of engagement within YD programs, as this construct may provide a rich characterization of young people's experiences and insights into how to sustain their active participation (Fredricks, 2011). 


\section{Engagement in Scoutreach}

Cognitive, emotional, and behavioral engagement

Bohnert et al. (2010) conceptualized engagement as comprised of three dimensions: cognitive, emotional, and behavioral. Cognitive engagement is investment in the learning process as well as how much youth value what they learn, and a commitment to mastering difficult skills (Bohnert et al., 2010; Li \& Lerner, 2013). Emotional engagement refers to positive and negative reactions toward program activities and may include enjoyment, enthusiasm, and feelings of belonging (Bohnert et al., 2010; Li \& Lerner, 2013). Behavioral engagement reflects both shallow (e.g., attendance) and deeper engagement (e.g., active participation, effort; Bohnert et al., 2010; Li \& Lerner, 2013). Scholars agree that engagement is a multifaceted dimension of youth participation that represents "the missing link in organized activity research" (Bohnert et al., 2010, p. 593).

\section{Present Study}

The present cross-sectional and mixed-methods study aimed to enrich understanding of the cognitive, emotional, and behavioral engagement of boys of color who participated in the Boston-area Scoutreach initiative. Scoutreach is an arm of BSA that aims to make Scouting more accessible to ethnically and racially diverse boys and young men in lower-resource communities (Wang et al., 2017). Our study was inspired by a longitudinal, mixed-methods investigation, the Character and Merit Project (CAMP; Hilliard et al., 2014). During focus groups in the CAMP study, Scoutreach leaders discussed challenges in trying to implement the standard Scouting program in a way that was functional, feasible, and engaging to Scouts who were primarily from urban, lower-resource communities (Hershberg et al., 2015). Those findings informed our focus on exploring facilitators of youth engagement in Scoutreach in greater depth.

We drew from prior research on youth engagement (e.g., Bohnert et al., 2010; Li \& Lerner, 2013) to assess cognitive, emotional, and behavioral engagement among ethnically and racially diverse boys from urban, lower-resource communities who participated in Scoutreach. We examined whether an adapted school engagement measure (Li \& Lerner, 2013) was appropriate for use in this YD program context. In addition, we analyzed interview data from Scoutreach leaders, parents/guardians, and youth to examine similarities and differences in their views of youth engagement in Scoutreach. We explored the following research questions:

1. Were Scouts cognitively, emotionally, and behaviorally engaged in Scoutreach?; 


\section{Engagement in Scoutreach}

2. What were the relations among dimensions of engagement and character attributes, self-perceived school competence, and intentional self-regulation?;

3. What were Scouts', parents/guardians', and leaders' views of how Scoutreach promoted youth engagement?; and

4. When interpreting the quantitative and qualitative data together, what types of metainferences can be made about youth engagement in Scoutreach and its relation to PYD?

\section{Method}

\section{Mixed-Methods Approach}

Mixed-methods approaches facilitate a richer and more holistic understanding of the potential processes through which involvement in programs may be linked to youth outcomes in comparison to the use of a single research method (Riggs \& Greenberg, 2004). Triangulation of data across sources and participant groups may be useful for assessing the validity of measures and for examining potential convergence and divergence in views (Guion, 2002; Hershberg et al., 2015). Accordingly, we collected quantitative and qualitative data from Scoutreach leaders, parents/guardians, and youth to examine similarities and differences in program-related experiences across data sources and participant groups.

\section{Quantitative Analysis}

We collected quantitative data from Scouts to assess whether dimensions of engagement were related to character attributes, self-perceived school competence, and intentional selfregulation. In light of the study's small sample, we imputed all missing values using SPSS Version 22. We performed 20 data imputations and, in SPSS, split the file by the imputation variable to generate pooled estimates (IBM SPSS Guide, 2011). We tabulated means and standard deviations and computed Pearson product-moment correlations.

\section{Qualitative Analysis}

We performed interpretative phenomenological analysis (IPA; Smith, 2011; Smith \& Osborn, 2008) of interview responses to examine the views and experiences of Scouts, parents/guardians, and leaders. This approach involves close, detailed analysis of individual cases, followed by analysis of patterns or themes across cases to assess potential convergence and divergence (Smith, 2011). There are several steps involved in IPA (Smith \& Osborn, 2008). 


\section{Engagement in Scoutreach}

After the first author developed initial codebooks for each participant group, she discussed the codes with two other trained qualitative researchers to validate her interpretations of participants' responses and to reach conceptual agreement on the codes. Next, using the initial codebooks, the first author and a second researcher independently coded approximately half of the transcripts in each participant group. They discussed their coding decisions and resolved any discrepancies (e.g., through adding codes or revising the definitions of codes). Kappa coefficients indicated adequate interrater reliability (.72 for Scouts, .73 for parents/guardians, and .75 for leaders).

After separate analyses of the quantitative and qualitative data, we examined areas of commonality across the three participant groups and across both sets of analyses. This concurrent triangulation approach (Creswell, 2009) helped to provide more in-depth information about participant experiences and outcomes, and may have offset the weaknesses associated with one method with the strengths associated with the other method.

\section{Participants}

We analyzed quantitative data from 32 Scouts (Mage $=9.97$ years, $S D=2.46$, Range $=6$ to 14) enrolled in two Scoutreach units in the greater Boston area. About a third $(31.3 \%)$ identified as Hispanic/Latino. The racial composition was 37.5\% multiethnic or multiracial, 15.6\% Black or African American, 9.4\% American Indian/Native American, 9.4\% Asian or Pacific Islander, 9.4\% White, and 6.3\% identified as Other. Qualitative data were collected from 10 Scouts (Mage $=11.40$ years, $S D=2.41$, Range $=6$ to 14 ).

We analyzed quantitative data from 32 parents/guardians (Mage $=40.0$ years, $S D=8.54$, Range $=26$ to 55). Half of the parents/guardians identified as Scouts' mothers. In regard to ethnicity, $31.3 \%$ of parents/guardians identified as Hispanic or Latino/a. The racial composition was $21.9 \%$ White, $18.8 \%$ Black or African American, $15.6 \%$ Other, $15.6 \%$ multiethnic or multiracial, $6.3 \%$ Asian or Pacific Islander, and 6.3\% identified as American Indian/Native American. Half of the parents/guardians had less than a Bachelor's degree and most (68.8\%) met United States Department of Housing and Urban Development criteria for low-income status. Qualitative data were collected from 10 parents/guardians (Mage $=37.0$ years, $S D=$ 9.37, Range $=27$ to 51 ).

Qualitative data were collected from five Scoutreach leaders ( $M_{\text {age }}=54.0$ years, $S D=10.83$, Range $=33$ to 62$)$. All were male, the majority $(60.0 \%)$ were White, and all had at least a 


\section{Engagement in Scoutreach}

Bachelor's degree. Their duration of involvement as leaders ranged from one year to approximately 20 years.

The size of the interview sample (i.e., 10 Scouts, 10 parents/guardians, and five leaders) was chosen with regard to the analytic approach that we used. IPA is recommended for use with small samples (e.g., Smith \& Osborn, 2008).

\section{Procedure}

This study was approved by the Tufts University Institutional Review Board. We also obtained a Certificate of Confidentiality from the National Institutes of Health.

Participants were recruited from two Scoutreach units in the Boston area. Scoutreach is headed by a District Executive, to whom the leaders report. The first author worked with the District Executive to select packs from whom to recruit participants, giving consideration to criteria such as convenience. According to the District Executive, the units that were selected were considered to be exemplary by the Council in light of their successful records of recruitment and retention. The first author attended unit meetings to describe the study to parents/guardians and to collect verbal consent and assent.

All data collection was completed during Scoutreach meetings. For questionnaire completion, Scouts received a small toy and parents/guardians received a $\$ 10$ gift card. All interview participants received a $\$ 15$ gift card. All interviews were transcribed by a professional transcription service or by the first author.

\section{Measures}

Scouts completed a modified version of the questionnaire used in the CAMP study (Hilliard et al., 2014), including measures of engagement, character attributes, self-perceived school competence, and intentional self-regulation (Wang et al., 2015a, 2015b). Items were scored using a five-point Likert-type scale ranging from 1 (Not at all like me) to 5 (Exactly like me); higher scores indicated greater endorsement of items. We created mean-level composite scores by averaging participants' responses across scale items. 


\section{Engagement in Scoutreach}

\section{Engagement}

Cognitive Engagement $(a=.95)$ was assessed using five items adapted from the school engagement measure used by Li and Lerner (2013; e.g., "I want to learn as much as I can in Scouts"). Emotional Engagement $(a=.79)$ was assessed using five items adapted from the school engagement measure (Li \& Lerner, 2013; e.g., "I think Scouts is fun and exciting").

Behavioral Engagement was assessed using five items adapted from the school engagement measure (Li \& Lerner, 2013; e.g., "I come to Scout meetings and activities on time"). Initially, scores showed low reliability $(a=.60)$. Based on analysis of inter-item correlations, we removed the first item ("I come to Scout meetings and activities prepared [with my uniform on, parent permission forms signed]"). Scores on the revised scale showed higher, albeit still relatively low, reliability $(a=.67)$.

\section{Character Attributes}

Character attribute items were from the Assessment of Character in Children and Early Adolescents (Wang et al., 2015a, 2015b). This measure assesses eight character-related attributes, some of which are reflected in the Scout Law ${ }^{1}$. We assessed seven character attributes. Obedience $(a=.73)$ was assessed using four items adapted from the conduct/behavior adequacy subscale of the Self-Perception Profile for Children (SPPC; Harter, 1982, 1983; e.g., "I act the way I am supposed to"). Religious Reverence was assessed using four items adapted from the Spiritual Transcendence Scale (Piedmont, 1999; e.g., "I like to read or listen to stories from my religion"). Scores showed low reliability $(a=.66)$, but analysis of inter-item correlations did not reveal any deletions that would enhance reliability. Cheerfulness ( $a=.74$ ) was assessed using three items adapted from the Positive and Negative Affect Scale for Children (Laurent, Potter, \& Catanzaro, 1994; e.g., "I am happy"). Kindness $(\mathrm{a}=.83)$ was assessed using four items adapted from the Caring scale in the 4-H Study of PYD (Lerner et al., 2005; e.g., "I'm kind to other kids"). Hopeful Future Expectations ( $a=.71$ ) was assessed using three items adapted from the Schmid \& Lopez (2011) scale (e.g., "I will have a happy family"). Trustworthiness $(a=.89)$ was assessed using five items adapted from the Personal Values subscale of the Character measure of PYD used in the 4-H Study (Lerner et al., 2005; e.g., "I can be counted on to tell the truth").

\footnotetext{
${ }^{1}$ Scout Law: A Scout is trustworthy, loyal, helpful, friendly, courteous, kind, obedient, cheerful, thrifty, brave, clean, and reverent (BSA, 2017).
} 


\section{Engagement in Scoutreach}

Helpfulness $(a=.72)$ was assessed using six items adapted from the Child Behavior Scale (Ladd \& Profilet, 1996; e.g., "I help people in my family").

\section{Self-Perceived School Competence}

Six items adapted from the academic competence subscale of the SPPC (Harter, 1982, 1983; e.g., "I am very good at my schoolwork") assessed self-perceived school competence. Scores showed low reliability $(a=.68)$. Analysis of inter-item correlations did not reveal any changes that would enhance reliability.

\section{Intentional Self-Regulation (ISR)}

Six items adapted from the selection, optimization, and compensation measure developed by Baltes and colleagues (e.g., Freund \& Baltes, 2002; e.g., "I am good at making plans") assessed ISR. Scores showed low reliability $(a=.63)$. Analysis of inter-item correlations did not reveal any changes to the scale that would enhance reliability.

\section{Parent/Guardian Demographic Questionnaire}

Parents/guardians completed 26 multiple-choice items regarding standard background information (e.g., education and income levels; Hilliard et al., 2014). The questionnaires were in English and Spanish.

\section{Youth Interview}

We used a modified version of the semi-structured interview protocol from the CAMP Study (Ferris, Hershberg, Su, Wang, \& Lerner, 2016) to ask youth about their experiences in Scouting. The protocol consisted of 18 items, including "How has Scouts affected you and your life?"

\section{Parent/Guardian Interview}

We used a semi-structured interview protocol to ask parents/guardians about their sons' involvement in Scouting. The protocol consisted of 16 items, including "What kind of an effect do you think that Scouts has on your son?" 


\section{Scoutreach Leader Interview}

We used a modified version of the semi-structured interview protocol used in the CAMP study (see Hilliard et al., 2014) to ask Scoutreach leaders about their views of Scouting. The protocol consisted of 16 items, including "How do you get boys engaged in Scoutreach?"

\section{Quantitative Results}

\section{Descriptive Analyses}

We calculated mean scores and standard deviations for the quantitative scales (Table 1). As expected, Scouts' average scores were moderate to high on dimensions of engagement, character attributes, self-perceived school competence, and intentional self-regulation.

Table 1. Scale Descriptive Statistics $(n=32)$

\begin{tabular}{|l|c|c|c|c|c|}
\hline \multicolumn{1}{|c|}{ Variable } & $\begin{array}{c}n \text { of } \\
\text { Items }\end{array}$ & $\begin{array}{c}\text { Mean (Standard } \\
\text { Deviation) }\end{array}$ & Min & Max & $\begin{array}{c}\text { Cronbach's } \\
\text { Alpha }\end{array}$ \\
\hline Cognitive engagement & 5 & $4.36(.93)$ & 1.0 & 5.0 & .95 \\
\hline Emotional engagement & 5 & $4.41(.62)$ & 2.8 & 5.0 & .79 \\
\hline Behavioral engagement* & 4 & $4.48(.57)$ & 4.13 & 4.75 & .67 \\
\hline Obedience & 4 & $3.88(.65)$ & 2.75 & 5.0 & .73 \\
\hline Religious reverence & 4 & $3.15(.91)$ & 1.5 & 5.0 & .66 \\
\hline Cheerfulness & 3 & $3.58(.95)$ & 1.67 & 5.0 & .74 \\
\hline Kindness & 6 & $4.06(.66)$ & 2.5 & 5.0 & .83 \\
\hline Hopeful future expectation & 3 & $4.44(.66)$ & 2.67 & 5.0 & .71 \\
\hline Trustworthiness & 5 & $3.76(.91)$ & 1.0 & 5.0 & .89 \\
\hline Helpfulness & 6 & $3.84(.63)$ & 2.5 & 5.0 & .72 \\
\hline Self-perceived school & 6 & $3.79(.69)$ & 2.5 & 4.83 & .68 \\
\hline Intentional self-regulation & 6 & $3.41(.62)$ & 2.5 & 4.5 & .63 \\
\hline
\end{tabular}

*Original behavioral engagement scale was comprised of five items. 
Engagement in Scoutreach

Table 2. Correlations among Dimensions of Engagement, Character Attributes, Self-Perceived School Competence, and Intentional Self-Regulation $(n=32)$

\begin{tabular}{|c|c|c|c|c|c|c|c|c|c|c|c|}
\hline & 1 & 2 & 3 & 4 & 5 & 6 & 7 & 8 & 9 & 10 & 11 \\
\hline \multicolumn{12}{|l|}{ 1. Obedience } \\
\hline 2. Reverence & .06 & & & & & & & & & & \\
\hline 3. Cheerfulness & $.45^{* *}$ & .17 & & & & & & & & & \\
\hline 4. Kindness & $.44^{*}$ & .05 & $.39 *$ & & & & & & & & \\
\hline 5. Hopeful future & $.41^{*}$ & .30 & .12 & $.58 * *$ & & & & & & & \\
\hline 6. Trustworthiness & $.71 * *$ & .03 & $.38^{*}$ & $.48^{* *}$ & $.57 * *$ & & & & & & \\
\hline 7. Helpfulness & $.65^{* *}$ & .06 & $.60 * *$ & $.78 * *$ & $.52 * *$ & $.66 * *$ & & & & & \\
\hline 8. School competence & $.51 * *$ & $.48^{* *}$ & .33 & $.52 * *$ & $.44^{*}$ & $.48^{* *}$ & $.51 * *$ & & & & \\
\hline 9. Intentional self-regulation & $.45^{* *}$ & $.39 *$ & $.36 *$ & $.60 * *$ & $.46 * *$ & $.48^{* *}$ & $.58 * *$ & $.63^{* *}$ & & & \\
\hline 10. Cognitive engagement & .26 & .16 & .34 & .25 & $.40 *$ & $.45^{*}$ & $.45^{*}$ & $.41^{*}$ & .24 & & \\
\hline 11. Emotional engagement & $.38^{*}$ & $.35^{*}$ & $.37 *$ & $.52 * *$ & $.43^{*}$ & $.41^{*}$ & $.69 *$ & $.59 *$ & $.48^{* *}$ & $.60 * *$ & \\
\hline 12. Behavioral engagement & .33 & .05 & .24 &. .28 & .33 & $.54 * *$ & .33 & .32 & .19 & $.77 * *$ & $.42 *$ \\
\hline
\end{tabular}

** Correlation is significant at the .01 level (2-tailed).

* Correlation is significant at the .05 level (2-tailed). 


\section{Engagement in Scoutreach}

\section{Scoutreach Engagement and Indicators of Healthy Youth Development}

We computed Pearson correlations to examine relations among all scales (Table 2). We interpreted the magnitudes of correlation coefficients using Cohen's guidelines (Hemphill, 2003). Cognitive engagement was moderately positively associated with hopeful future expectation, trustworthiness, helpfulness, and self-perceived school competence. Emotional engagement was moderately to highly positively associated with obedience, reverence, cheerfulness, kindness, hopeful future expectation, trustworthiness, helpfulness, self-perceived school competence, and ISR. Behavioral engagement was positively associated with trustworthiness, with a large magnitude.

\section{Qualitative Results}

\section{Perceived Aspects of Scoutreach Linked to Cognitive Engagement}

Leaders described how Scouts were cognitively engaged, or how they tried to promote cognitive engagement, in Scoutreach. Three (60.0\%) leaders suggested that they fostered Scouts' cognitive engagement by exposing them to mentally challenging experiences and acknowledging their mastery of difficult tasks and skills: "What I think [Scouts] like about [the activity] is it's challenging"; "Get [Scouts] outdoors in challenging situations. Congratulate them when they do it"; and "It's the challenge, and what we have is the biggest classroom in the world. Just keep pushing the envelope." Two (40.0\%) leaders described promoting cognitive engagement during Scout meetings through presenting lessons concisely and interactively: "In the meetings . . . you're learning while you're having fun" and

The Boy Scout meeting is a 90-minute meeting and the way we try and do it is so they're broken up into small blocks which will keep young boys' attention, 15-20 minutes on one thing. We try to use a combination of a teaching section where they work on a Scout skill, a planning section where they think about assuming responsibility . . . some physical activity.

Similarly, three (30.0\%) parents/guardians described the importance of teaching Scouts interesting, useful, and important information and mentioned that their sons felt proud when they accomplished important tasks: "[Scouting] offers not only the theory of learning . . . but they do things that are practical and they prove themselves"; "You've got to make [Scouting] 


\section{Engagement in Scoutreach}

interesting for [youth] so they're willing to participate"; and "[My son's] proud of himself when he's ... learned a new task and he's completed it."

Half $(50.0 \%)$ of Scouts said that they thought the skills that they learned in Scoutreach were useful and important: "[first-aid] is important to learn because you can help people that's hurt" and "I like to learn new things . . . learning about new knots, new first-aid type things . . . like how to make splints and stuff. I think it's really cool and it's really useful information." One Scout $(10.0 \%)$ described how he personally valued what he learned in Scouting: "For me, it was extremely rewarding to learn how to use axes, knives, make fire, and also how to make shelters." Two Scouts (20.0\%) also described feeling proud of their learning and accomplishments in Scouting (e.g., through earning merit badges).

\section{Perceived Aspects of Scoutreach Linked to Emotional Engagement}

All five $(100.0 \%)$ leaders discussed the importance of exposing Scouts to fun and exciting activities to foster emotional engagement: "At the Cub Scout level . . . do stuff that's exciting. That's number one"; "You've got to make it fun. This is where we probably have the hardest time because we're trying to follow the curriculum, but . . . you've got to have a fun element"; and "The children that really get into the outdoor parts of the program tend to stay because they love the outdoors program so much that they want to continue." One (20.0\%) leader linked Scouts' positive affect toward Scouting to receiving recognition for achievements: "They need to know they're earning things. Nothing makes a child prouder and more wanting to come in than when you call them to the front of the room once a month and you hand them a belt loop."

Four (40.0\%) parents/guardians suggested how their sons' emotional engagement was linked to Scoutreach activities; in particular, camping: "[Camping] was nice and [my son] really enjoyed it"; "[My son] loves archery. He loves fishing. He loves the activities where he can do them outside of Scouts as well"; "I think [the activities] are enjoyable for [my son]"; and "[My son] had a lot of fun [at camp]." More than half (60.0\%) of parents/guardians also suggested that their sons' emotional engagement was linked to their relationships with other boys in Scoutreach. For example, "He's excited to come. He knows he's going to ... . [interact] with other boys" and "He likes being around his friends."

For four (40.0\%) Scouts, emotional engagement was linked to relationships. For instance, "I think [Scouting] is fun because there's other people," and "[I feel] happy [at Scouting] 


\title{
Engagement in Scoutreach
}

because you get to meet your friends that you have there." For most (70.0\%) Scouts, emotional engagement was related to different activities. For example, "[Scouting has] made me feel more ... y you go outside more, so you feel different sometimes"; "I feel happy, excited [at Scouting] . . . because I don't know what we're gonna do next"; "I enjoy [Scouting] and I feel happy when I'm here. It's another thing I do instead of just staying home all day"; and "We don't just learn, we have fun sometimes, playing dodge ball and stuff like that."

\section{Perceived Aspects of Scoutreach Linked to Behavioral Engagement}

All five (100.0\%) leaders described the importance of getting Scouts actively involved in memorable activities to promote behavioral engagement: "Get them out early, in activities that they will remember for the rest of their Scouting career - BBs, archery, out to a camp . . . once you set that hook, they're always going to want to do more"; "Do a campfire, have some s'mores, and then let them go back on Monday and the first thing out of their mouth when they walk in the classroom is 'I built a fire on Saturday.' That's what works."; "You have to have a physical element that is active"; and "Do things. Get the boys hands-on experience."

Two (20.0\%) parents/guardians also described their sons' effort and active participation in different activities: "[Scouting] worked with him and he sees how much fun he can have outside doing things" and

\begin{abstract}
When he went to the Pumpkin Fest . . . he didn't want to do the bow and arrow. He said 'I'm not going to be able to do it.' Even though he didn't really make the shot . . . he tried it. He liked the fact that he's able to do that.
\end{abstract}

In addition, all (100.0\%) Scouts described their effort and active participation in camping and other activities: "we . . . make a fire and sleep in tents that we pitched"; "[at summer camp], we go swimming, archery, rifle shooting, and more"; "when we went to the camps, we did archery, rock climbing, BB guns, and how to make a fire"; "I am actually helping staff the youth leadership training over the summer"; and "my last major [service project] was actually cleaning up the garden cemetery." Some Scouts described, more generally, why they were behaviorally engaged in Scoutreach, as compared to other activities: "I think Scouts is better [than basketball] because you get to do more things" and "At home, I would just play games all night, but at Boy Scouts, I'm active, like how I'm supposed to be" 


\section{Engagement in Scoutreach}

\section{Summary of Mixed-Methods Findings}

Questionnaire data suggested that youth were engaged cognitively, emotionally, and behaviorally, with nuanced relations among dimensions of engagement, character attributes, self-perceived school competence, and intentional self-regulation. Emotional engagement, or having a positive affect toward Scoutreach, seemed to be a particularly important dimension of engagement that was related to all of the indicators of PYD.

In interviews, leaders, parents/guardians, and Scouts described positive views of Scoutreach. However, the groups differed in their emphases on key facets of Scoutreach linked to youth engagement. Whereas leaders and Scouts primarily described Scouts' experiences in camping and other outdoor activities as particularly important in promoting boys' meaningful and sustained participation in Scoutreach, parents/guardians more strongly emphasized the importance of relationships that their sons formed with their leaders and other Scouts.

On the questionnaires, Scouts had high average scores on the dimensions of engagement that we assessed. The interviews expanded on the quantitative findings by elucidating which aspects of Scoutreach were most strongly related to boys' engagement. For example, participants commonly linked Scouts' engagement in Scoutreach to outdoor activities and the opportunity to build strong relationships with leaders and other Scouts.

Analyses also converged to suggest the important role of emotional engagement. Questionnaire data suggested that emotional engagement was positively associated with the most indicators of PYD, and was uniquely associated with several of these indicators. Similarly, in interviews, leaders, parents/guardians, and Scouts provided vivid examples of how Scouts were emotionally engaged, as compared to how they demonstrated other dimensions of engagement. Interviews with leaders, in particular, seemed to suggest that emotional engagement helped to promote cognitive and behavioral engagement.

\section{Discussion}

Research suggests that, although it may be difficult for some youth to navigate experiences associated with living in lower-resource communities, there are youth who thrive despite their exposure to adversity (Jain, Buka, Subramanian, \& Molnar, 2012). High-quality YD programs have the potential to maximize diverse young people's potential for thriving by embracing and enhancing their individual strengths and buffering them against contextual risk factors (Urban 


\section{Engagement in Scoutreach}

et al., 2009). However, more research is needed to better understand how such programs effectively engage youth (Bohnert et al., 2010). We used cross-sectional, mixed-methods data to examine how boys of color were cognitively, emotionally, and behaviorally engaged in Scoutreach, and whether these dimensions of engagement were related to character attributes, self-perceived school competence, and intentional self-regulation.

Scouts had high average scores on the dimensions of engagement and moderate to high average scores on character attributes, consistent with prior research with Scouting (Lynch et al., 2016; Wang et al., 2015b). Quantitative data suggested nuanced relations among dimensions of engagement and character attributes. These results also aligned with prior research linking dimensions of youth engagement and involvement in programs to indicators of positive development (Ramey et al., 2015; Tiffany et al., 2012).

Scoutreach leaders and Scouts primarily linked camping and outdoor activities to all dimensions of engagement, whereas parents/guardians primarily linked Scouts' emotional engagement, in particular, to relationships with other boys. These data were consistent with prior work suggesting that the perceptions of practitioners, parents/guardians, and youth may vary in regard to what constitutes youth thriving, and barriers to thriving, within the context of YD programs (King et al., 2005).

\section{Camping and the Outdoors}

In interviews, leaders, parents/guardians, and Scouts all acknowledged the importance of Scouts' involvement in camping and outdoor activities. Based on descriptions from Scouts and leaders, camping appeared to be the facet of Scoutreach most strongly related to Scouts' interest in joining and their sustained engagement.

A large body of research suggests the developmental benefits associated with camping and other forms of experiential learning (Henderson et al., 2007; Thurber, Scanlin, Scheuler, \& Henderson, 2007), including positive identity, independence, social skills, positive values and decision-making, and environmental awareness and physical skills (Thurber et al., 2007). Camping may be an especially meaningful experience for youth who lack access to supportive adults (Henderson et al., 2007) or for youth from urban, lower-resource communities, and may function as an intervention setting through cultivating strong relationships and promoting skill building (Bialeschki, Henderson, \& James, 2007). Scoutreach leaders and Scouts described 


\section{Engagement in Scoutreach}

camping and other outdoor activities as promoting these assets and as helping to facilitate the application of learned attributes and skills.

\section{Potential Implications for Youth Programming}

Our findings have important implications for how Scoutreach leadership, and leaders of similar YD programs, recruit participants, implement their programming, and continue to examine the experiences and outcomes of diverse youth. First, the present study reinforced the importance of capturing the views and experiences of different stakeholders (i.e., leaders, parents/guardians, and youth). There were nuances, for instance, in which stakeholders linked aspects of Scoutreach to youth engagement. These findings may have important implications for how Scoutreach leadership, and similar YD programs, markets its programming. For instance, in describing the initiative to prospective Scouts, leaders may want to continue to strongly emphasize the opportunities to gain exposure to new experiences, namely camping and the outdoors. In comparison, when describing the initiative to parents/guardians, leaders may want to emphasize how Scoutreach helps to cultivate supportive interpersonal relationships.

Findings highlighted the potential developmental benefits associated with immersing underrepresented youth in experiential learning opportunities in outdoor contexts to which they otherwise would not have had access. Thus, as part of efforts to effectively recruit, engage, and sustain youth participants, Scouting and similar YD programs should explore ways to continue to involve these youth in new and exciting experiences which, as one Scoutreach leader stated, "Get [youth] out of the neighborhood, and let them see and experience other things that kids in different socioeconomic circumstances take for granted."

\section{Potential Implications for Future Research}

Findings also raised potential implications for future research with Scoutreach and similar YD programs. Follow-up longitudinal work should address in greater depth the potential developmental impacts of the camping experience. This research might examine topics such as variation in the amount of emphasis that Scoutreach units place on camping and how the camping-related views and experiences of Scoutreach youth compare to those of Scouts in the traditional Scouting program. Such research may, again, also have important implications for how Scoutreach is marketed. 


\section{Engagement in Scoutreach}

As noted by King et al. (2005), considerable variation in perspectives may exist across communities and cultural groups. Although we examined the perspectives of an ethnically, racially, and economically diverse sample, between-group comparisons were beyond the scope of our study. Thus, future research may want to examine whether individuals' views of, and experiences in, Scoutreach (and BSA) vary across communities and cultural groups.

Future research should also examine the role of parent/guardian involvement promoting youth engagement, and potential barriers and facilitators to their involvement. In the present study, leaders described parent/guardian involvement as essential for Scouts' success, but also acknowledged challenges associated with promoting their participation. Research should consider how parents/guardians demonstrate involvement (e.g., attending meetings, participating in activities, serving as leaders), and examine whether differences exist in relation to youth outcomes.

\section{Limitations and Additional Future Directions}

A primary limitation of our study was its small sample. Future research should examine potential differences between participating and non-participating units in Scoutreach and other YD programs based on analysis of administrative data, such as youth and leader retention rates or program benchmarks or milestones completed by units.

Our cross-sectional design precluded assessment of potential intra-individual variability. Although RDS metatheory informed this investigation and our interpretation of the results, this perspective involves individual-context relations across time and place, which could not be tested using a cross-sectional design. Thus, future longitudinal research should examine whether young people's Scoutreach-related experiences and outcomes inform their subsequent individual-context developmental regulations. Directional hypotheses should be tested, in light of our findings. For instance, leaders seemed to suggest that Scouts' emotional engagement, or employing an "emotional hook," helped to promote their cognitive and behavioral engagement in Scoutreach. This idea should be tested in subsequent research.

We collected data only from self-selected individuals affiliated with a specific YD initiative in a particular geographic area, thus limiting the generalizability of findings. In addition, Scouts enrolled in Scoutreach may differ in important ways from their uninvolved peers. Thus, future research should collect data from demographically similar comparison samples. 


\section{Engagement in Scoutreach}

The study also contained threats to internal validity and reliability. For instance, there may have been issues associated with how the dimensions of engagement were operationalized. Future research should more rigorously examine the validity of these scales by performing exploratory and confirmatory factor analyses.

Characteristics associated with the researchers who conducted the interviews might also have impacted the validity of the data. Research on conducting qualitative studies suggests the importance of forming positive relationships with study participants, in particular members of marginalized populations, to help break down communication and trust barriers and to gain an insider's perspective on participants' experiences (Dwyer \& Buckle, 2009).

Despite these limitations, the present study helps to expand developmental scholarship by examining the experiences of urban boys of color who participated in an arm of one of the oldest and largest youth-serving programs in the country. The mixed-methods research design supported a multifaceted understanding of which aspects of Scoutreach were linked to youth engagement. Although this study is an initial, exploratory attempt to examine in more depth the experiences of urban boys of color within a particular OST context, the findings raise important questions and considerations for future research and practice.

\section{Conclusions}

High-quality YD programs have the potential to function as key contexts in the lives of ethnically, racially, and economically diverse youth; in particular, youth from lower-resource communities (Francois et al., 2011). In-depth research is needed that examines the experiences of these youth and different stakeholders as part of efforts to enhance the quality of young people's experiences across OST settings. We aimed to enhance understanding of the phenomenology of the experiences of boys of color within a specific OST context, Scoutreach, and sought to elucidate how their experiences within this setting related to their engagement in the initiative and indicators of PYD (e.g., character attributes). Future research with Scoutreach and other programs that serve demographically similar youth may reveal greater insights into the developmental processes involved in youth program engagement. 


\section{Acknowledgements}

The preparation of this article was supported, in part, by a dissertation award from the Society for Research in Child Development awarded to the first author. The first author would like to thank members of her dissertation committee: Richard M. Lerner (Chair), Ellen E. Pinderhughes, and Michael Cunningham. She would also like to thank her colleagues from the Institute for Applied Research in Youth Development at Tufts University; in particular, Shaobing Su, Kaitlyn A. Ferris, and Jun Wang.

\section{References}

Bialeschki, M. D., Henderson, K. A., \& James, P. A. (2007). Camp experiences and developmental outcomes for youth. Child and Adolescent Psychiatric Clinics of North America, 16(4), 769-788.

Bohnert, A., Fredricks, J., \& Randall, E. (2010). Capturing unique dimensions of youth organized activity involvement: Theoretical and methodological considerations. Education \& Educational Research, $80(4), 576-610$.

Boy Scouts of America (BSA). (2017). Retrieved from http://www.scouting.org/

Creswell, J. W. (2009). Research design: Qualitative, quantitative, and mixed methods approaches. Thousand Oaks, CA: SAGE.

Dwyer, S. C., \& Buckle, J. L. (2009). The space between: On being an insider-outsider in qualitative research. International Journal of Qualitative Methods, 8(1), 54-63.

Ferris, K. A., Hershberg, R. M., Su, S., Wang, J., \& Lerner, R. M. (2016). Character development among youth of color from low-SES backgrounds: An examination of Boy Scouts of America's ScoutReach program. Journal of Youth Development, 10(3), 14-30.

Francois, S., Overstreet, S., \& Cunningham, M. (2011). Where we live: The unexpected influence of urban neighborhoods on the academic performance of African American adolescents. Youth \& Society, 42(2), 1-22.

Fredricks, J. A. (2011). Engagement in school and out-of-school contexts: A multidimensional view of engagement. Theory Into Practice, 50(4), 327-335.

Freund, A. M., \& Baltes, P. B. (2002). Life-management strategies of selection, optimization, and compensation: Measurement by self-report and construct validity. Journal of Personality and Social Psychology, 82(4), 642-662.

Guion, L. A. (2002). Triangulation: Establishing the validity of qualitative studies. University of Florida, Institute of Food and Agricultural Sciences. Retrieved from http://sites.duke.edu/niou/files/2014/07/W13-Guion-2002-Triangulation-Establishing-the-Validityof-Qualitative-Research.pdf

Harter, S. (1982). The perceived competence scale for children. Child Development, 53(1), 87-97. 


\section{Engagement in Scoutreach}

Harter, S. (1983). Developmental perspectives on the self-system. In M. Heatherington (Ed.), Handbook of child psychology: Social and personality development (Vol. 4). New York, NY: Wiley.

Hemphill, J. F. (2003). Interpreting the magnitudes of correlation coefficients. American Psychologist, $58(1), 78-80$.

Henderson, K. A., Bialeschki, M. D., Scanlin, M. M., Thurber, C., Whitaker, L. S., \& Marsh, P.E. (2007). Components of camp experiences for positive youth development. Journal of Youth Development, $1(3), 15-26$.

Hershberg, R. M., Chase, P. A., Champine, R. B., Hilliard, L. J., Wang J., \& Lerner, R. M. (2015). "You can quit me, but I'm not gonna quit you": A focus group study of leaders' perceptions of their positive influences on youth in Boy Scouts of America. Journal of Youth Development, 10(2), 5-30.

Hilliard, L. J., Hershberg, R. M., Wang, J., Bower, E. P., Chase, P. A., Champine, R. B., . . Lerner, R. M. (2014). Program innovations and character in Cub Scouts: Findings from year 1 of a mixedmethods, longitudinal study. Journal of Youth Development, 9(4), 6-30.

IBM SPSS missing values 20. (2011). Retrieved from https://www.csun.edu/sites/default/files/missing-values20-64bit.pdf

Jain S., Buka S. L., Subramanian S. V., \& Molnar, B. E. (2012). Protective factors for youth exposed to violence: Role of developmental assets for building emotional resilience. Youth Violence and Juvenile Justice, 10(1), 107-129.

King, P. E., Dowling, E. M., Mueller, R. A., White, K., Schultz, W., Osborn, P., . . Scales, P. C. (2005). Thriving in adolescence: Voices of youth-serving practitioners, parents, and early and late adolescents. Journal of Early Adolescence, 25(1), 94-112.

Ladd, G. W., \& Profilet, S. M. (1996). The Child Behavior Scale: A teacher-report measure of young children's aggressive, withdrawn, and prosocial behaviors. Developmental Psychology, 32(6), 1008-1024.

Lastovicka, J. L., Bettencourt, L. A., Hughner, R. S., \& Kuntze, R. J. (1999). Lifestyle of the tight and frugal: Theory and measurement. Journal of Consumer Research, 26(1), 85-98.

Laurent, J., Potter, K., \& Catanzaro, S. J. (1994). Assessing positive and negative affect in children: The development of the PANAS-C. Paper presented at the 26th annual Convention the National Association of School Psychologists. Seattle, WA.

Lee, S. A., Borden, L. M., Serido, J., \& Perkins, D. F. (2009). Ethnic minority youth in youth programs: Feelings of safety, relationships with adult staff, and perceptions of learning social skills. Youth \& Society, 41(2), 234-255.

Lerner, R. M., Agans, J. P., DeSouza, L. M., \& Gasca, S. (2013). Describing, explaining, and optimizing within-individual change across the life span: A relational developmental systems perspective. Review of General Psychology, 172), 179-183. 


\section{Engagement in Scoutreach}

Lerner, R. M., Lerner, J. V., Almerigi, J., Theokas, C., Phelps, E., Gestsdóttir, S., . . von Eye, A. (2005). Positive youth development, participation in community youth development programs, and community contributions of fifth grade adolescents: Findings from the first wave of the 4-H Study of Positive Youth Development. Journal of Early Adolescence, 25(1), 17-71.

Li, Y., \& Lerner, R. M. (2013). Interrelations of behavioral, emotional, and cognitive school engagement in high school students. Journal of Youth and Adolescence, 42(1), 20-32.

Lynch, A. D., Ferris, K. A., Burkhard, B., Wang, J., Hershberg, R. M., \& Lerner, R. M. (2016). Character development within youth development programs: Exploring multiple dimensions of activity involvement. American Journal of Community Psychology, 57(1-2), 73-86.

Overton, W. F. (2015). Process and relational developmental systems. In W. F. Overton \& P. C. Molenaar (Eds.) (Editor-in-Chief: R. M. Lerner), Handbook of child psychology and developmental science. Vol. 1: Theory and method (7th ed., pp. 9-62). Hoboken, NJ: Wiley.

Overton, W. F., \& Lerner, R. M. (2014). Fundamental concepts and methods in developmental science: A relational perspective. Research in Human Development, 11(1), 63-73.

Piedmont, R. L. (1999). Does spirituality represent the sixth factor of personality? Spiritual transcendence and the five-factor model. Journal of Personality, 676), 985-1013.

Ramey, H. L., Rose-Krasnor, L., Busseri, M. A., Gadbois, S., Bowker, A., \& Findlay, L. (2015). Measuring psychological engagement in youth activity. Journal of Adolescence, 45, 237- 249.

Riggs, N. R., \& Greenberg, M. T. (2004). After-school youth development programs: A developmentalecological model of current research. Clinical Child and Family Psychology Review, 73), 177-190.

Roth, J. L., \& Brooks-Gunn, J. (2016). Evaluating youth development programs: Progress and promise. Applied Developmental Science, 20(3), 188-202.

Schmid, K. L., \& Lopez, S. (2011). Positive pathways to adulthood: The role of hope in adolescents' constructions of their futures. In R. M. Lerner, J. V. Lerner, \& J. B. Benson (Eds.), Advances in child development and behavior, Vol. 41: Positive youth development (pp. 72-89). London, England: Academic Press.

Smith, J. A. (2011). Evaluating the contribution of interpretative phenomenological analysis. Health Psychology Review, 5(1), 9-27.

Smith, J. A., \& Osborn, M. (2008). Interpretative phenomenological analysis. In J. A. Smith (Ed.), Qualitative psychology. Thousand Oaks, CA: SAGE.

Thurber, C. A., Scanlin, M. M., Scheuler, L., \& Henderson, K. A. (2007). Youth development outcomes of the camp experience: Evidence for multidimensional growth. Journal of Youth and Adolescence, 36(3), 241-254.

Tiffany, J. S., Exner-Cortens, D., \& Eckenrode, J. (2012). A new measure for assessing youth program participation. Journal of Community Psychology, 40(3), 277-291. 


\section{Engagement in Scoutreach}

Urban, J. B., Lewin-Bizan, S., \& Lerner, R. M. (2009). The role of neighborhood ecological assets and activity involvement in youth developmental outcomes: Differential impacts of asset rich and asset poor neighborhoods. Journal of Applied Developmental Psychology, 30(5), 601-614.

Vandell, D. L., Larson, R. W., Mahoney, J. L., \& Watts, T. W. (2015). Children's organized activities. In M. H. Bornstein \& T. Leventhal (Eds.) (Editor-in-Chief: R. M. Lerner), Handbook of child psychology and developmental science. Vol. 4: Ecological settings and processes in developmental systems (7th ed., pp. 305-344). Hoboken, N.J.: Wiley.

Wang, J., Champine, R. B., Ferris, K.A., Hershberg, R. M., Warren, D. J., Burkhard, B. M., . . Lerner, R. M. (2017). Is the Scoutreach initiative of Boy Scouts of America linked to character development among socioeconomically, racially, and ethnically diverse youth?: Initial explorations. Journal of Youth and Adolescence. doi: 10.1007/s10964-017-0710-8

Wang, J., Ferris, K. A., Hershberg, R.M., \& Lerner, R.M. (2015a). Developmental trajectories of youth character: A five-wave longitudinal study of Cub Scouts and non-Scout boys. Journal of Youth and Adolescence, 44(12), 2359-2373.

Wang, J., Hilliard, L. J., Hershberg, R. M., Bowers, E. P., Chase, P. A., Champine, R. B., . . Lerner, R. M. (2015b). Character in childhood and early adolescence: Models and measurement. Journal of Moral Education, 44(2), 165-197. 